was born in the Netherlands. The HIV incidence in the time period 2005-2009, as well as the overall HIV incidence in the total study period, was four times higher in the HIV-PEP cohort compared to the ACS (Abstract O1-S10.01 table 1). In the HIV-PEP cohort the overall HIV incidence was 6.49 (95\% CI 3.24\% to $11.62 \%$ ) per 100 PY, compared to 1.59 (95\% CI $1.25 \%$ to $2.0 \%$ per 100 PY in the MSM in the ACS in the same study period $(p<0.001)$.

Conclusions The HIV incidence of MSM who were prescribed a PEP course was four times higher than in the overall gay community in Amsterdam. Our results show that PEP users belong to a group of MSM at high risk for HIV infection. Further analyses will be conducted to compare predictors of HIV seroconversion in both cohorts.

\section{1-S10.02 HIV AND BACTERIAL STI TESTING AMONG MEN WHO HAVE SEX WITH MEN IN THE USA}

doi:10.1136/sextrans-2011-050109.56

1J G Rosenberger, ${ }^{1} \mathrm{~B}$ Van Der Pol, ${ }^{1} \mathrm{D}$ Herbenick, ${ }^{2} \mathrm{D} S$ Novak, ${ }^{1} \mathrm{M}$ Reece, ${ }^{3} \mathrm{~J} D$ Fortenberry. ${ }^{1}$ Indiana University, Bloomington, USA; ${ }^{2}$ Online Buddies Inc, Cambridge, USA; ${ }^{3}$ Indiana University, School of Medicine, Indianapolis, USA

Purpose Growing literature has documented the extent to which bacterial sexually transmitted infections (STI) facilitate transmission/acquisition of HIV. Due to the disproportionate impact of HIV on men who have sex with men (MSM), public health messaging encourages routine STI and HIV screening among this population. This study sought to document testing behaviours among MSM in the US and understand factors associated with STI testing.

Methods Data were collected via an internet survey from 27756 18-80 year old MSM members of an online sexual networking website. Measures included sociodemographics, STI/HIV testing behaviours, STI/HIV diagnoses, and lifetime sexual behaviour history. Results Participants' mean age was 39.0 years, ethnicities included white (84.5\%), Latino (6.4\%), African American (3.5\%), and most $(79.9 \%)$ identified as homosexual. Over half $(54.2 \%)$ indicated they were not in a romantic relationship, and nearly all (88.9\%) had been sexually active with more than one partner in the past year. Testing rates (past year) were similar for both $\operatorname{HIV}(65.4 \%)$ and STI (60.8\%), yet testing in the past 6 months differed with men reporting more frequent STI testing (63.8\%) compared to HIV (46.5\%); rates of infection in the previous 2 years included gonorrhoea (4.5\%), Chlamydia $(4.1 \%)$, and HIV (3.2\%), with lifetime HIV infection rate being $12.5 \%$. Among men living with HIV infection, rates of bacterial STI testing in the past year were higher $(76.1 \%)$, with gonorrhoea and Chlamydia rates being $8.6 \%$ and $7.6 \%$ respectively. Both race and sexual orientation were predictive of bacterial STI testing in the past year, with non-white and bisexual men being less likely to have done so $(p<0.01)$.

Conclusions These data provide a large scale assessment of HIV and STI testing among MSM in the US Findings from this study highlight gaps in screening between bacterial STI and HIV and suggest a need to promote services that offer HIV and STI testing simultaneously.

\section{1-S10.03 LYMPHOGRANULOMA VENEREUM, HIV AND HIGH-RISK BEHAVIOUR: FINDINGS FROM LGV ENHANCED SURVEILLANCE AND MATHEMATICAL MODELLING}

doi:10.1136/sextrans-2011-050109.57

${ }^{1} \mathrm{M}$ Ronn, ${ }^{1} \mathrm{G}$ Garnett, ${ }^{2} \mathrm{G}$ Hughes, ${ }^{2} \mathrm{C}$ Ison, ${ }^{2} \mathrm{~S}$ Simms, ${ }^{2} \mathrm{~S}$ Alexander, ${ }^{1} \mathrm{H}$ Ward. ${ }^{1}$ Imperial College London, London, UK; ${ }^{2}$ Health Protection Agency, UK

Background Lymphogranuloma venereum (LGV) re-emerged in industrialised countries in the early 2000s and most transmission is assumed to occur among high-risk HIV-positive men who have sex with men (MSM). As the epidemic matures, we would expect to see declining HIV prevalence among cases as the transmission moves outside the initial core groups, however LGV cases continue to present with high levels of HIV-positivity. We have previously conducted a systematic review and a meta-analysis of literature published on LGV and found an eightfold greater likelihood of HIV-positivity among LGV cases when compared to controls with non-LGV chlamydia. In this study we investigate the role of high-risk behaviour serosorting and differences in transmission probability as contributing factors to the persistent association between HIV and LGV.

Methods Data from LGV Enhanced Surveillance in the UK were used to investigate the relationship between HIV-positivity and LGV acquisition by setting HIV-positivity as the outcome in logistic regression. A simple deterministic compartmental model was developed, stratified by HIV-status and risk behaviour, to simulate the spread of infection among MSM in a large metropolitan population. Assortative mixing for HIV status and high-risk behaviour were controlled independently of each other see Abstract O1-S10.03 figure 1

Results Up to date approximately 81\% of the 1500 confirmed LGV cases have had surveillance forms returned with above $70 \%$ HIVprevalence among cases over time. HIV-positive LGV cases were more likely to report unprotected anal intercourse and greater number of partners than HIV-negative LGV cases in surveillance data. The mathematical model indicates that extreme levels of serosorting would be needed to explain the high prevalence of HIV in the presence of high-risk HIV-negative group. Increase in the number of HIV-positive individuals, higher transmission probability in HIV-positive and serosorting together were better able to reproduce the strong association between HIV and LGV.

Conclusions Differential acquisition of LGV occurs according to HIV status. LGV acquisition among HIV-positives may be due to serosorting together with an increased transmission probability due to reduced condom use and sexual practices, and potential biological susceptibility. Findings suggest that HIV-positive MSM need targeted health promotion in view of their different risk behaviours.
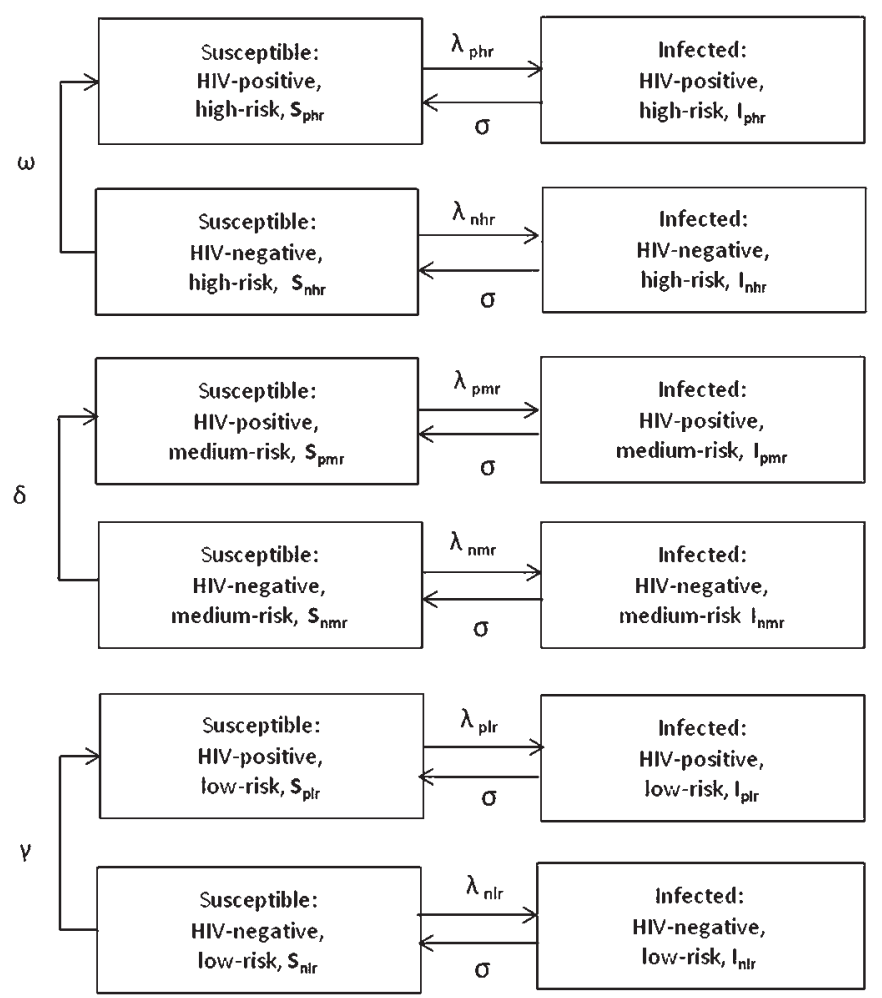

Abstract 01-S10.03 Figure 1 Flowchart illustrating the model structure. 